\title{
THE PRICE-VOLATILITY FEEDBACK RATE: AN IMPLEMENTABLE MATHEMATICAL INDICATOR OF MARKET STABILITY
}

\author{
EMILIO BARUCCI \\ Dipartimento di Statistica e Matematica Applicata all'Economia, Università di Pisa \\ Paul Malliavin \\ Académiedes Sciences, Paris \\ MARIA ElVIRA MANCINO \\ DIMAD, Università di Firenze \\ ROBERTO RENÒ \\ Dipartimento di Economia Politica, Università di Siena \\ Anton Thalmaier \\ Institut für Angewandte Mathematik, Universität Bonn
}

\begin{abstract}
Geometric analysis of iterated cross-volatilities of asset prices is adopted to assess the stability of the (risk-free) measure under infinitesimal perturbations. Perturbations of asset prices evolve through time according to an ordinary linear differential equation (hedged transfer). The decay (feedback) rate is explicitly computed through a Fourier series method implemented on high frequency time series.
\end{abstract}

KEY WoRDs: volatility matrix, market stability, liquidity, Greeks, Fourier series, stochastic calculus of variations, moving frame

\section{INTRODUCTION}

Feedback effects of asset prices on volatilities are recognized in the financial markets literature both theoretically and empirically. Lack of liquidity, related to asset price movements, induces erratic motion of the asset price and therefore amplifies its volatility: Trading by a large trader or by an agent employing a portfolio insurance-hedging strategy can originate a feedback effect of the asset price on its volatility with destabilizing effects; on the other hand, there are traders (fundamentalists and arbitrageurs) who trade in order to exploit perturbations and therefore dampen them (Friedman's conjecture; see Friedman 1953).

In this paper we will construct a measure of these effects and we will show how to compute it. Our setting is very general, our main assumption will be of qualitative nature

Address correspondence to Dr. Anton Thalmaier, Institut für Angewandte Mathematik, Universität Bonn, Wegelerstr. 6, 53115 Bonn, Germany; e-mail: anton@wiener.iam.uni-bonn.de. 
and model free: we shall assume that instantaneous volatilities are functions of price levels, these functions being time dependent and unknown; the only assumption made is that they are of class $C^{2}$.

Our setting includes stochastic volatility as well as level dependent volatility models; we shall not assume market completeness.

Working under this general assumption, we shall produce a time-dependent elasticity matrix, implementable in real time from asset price observations. Eigenfunctions associated with positive eigenvalues of the elasticity matrix correspond to instability directions of the market; eigenfunctions associated with negative eigenvalues correspond to stability directions of the market.

The key mathematical tool to construct the elasticity matrix is a new methodology of transferring price perturbations through time, using inertial frame transport; this transport is designed so that through time the variation is governed by a first-order linear ordinary differential equation. It is well known that perturbations' evolution through time is governed by a linear stochastic differential equation; in some sense it can be said that our inertial frame transport approach allows us to partially eliminate that stochasticity (hedged transfer). The rate of variation through time of the initial perturbation is given by the elasticity matrix. The method can be developed for any finite number of assets. In this paper we shall deal with the univariate and the bivariate cases; the general $n$-variate case needs more lengthy computations and will be treated in a subsequent paper.

Volatility is a problem in financial markets for many reasons. First, it is very difficult to measure; volatility of a diffusion process is well defined in terms of the quadratic variation, but then a finite sample of observations is used to compute it. Various methodologies can be adopted to this end but volatility estimate is sensitive to the methodology adopted and biases can be introduced - in particular in the handling of high frequency data (e.g., see Andersen and Bollerslev 1998; Barucci and Renò 2000, 2002). Financial time series volatility shows patterns' regularities that are hard to model and to justify theoretically. A partial list of stylized facts includes: volatility varies over time with a strong autoregressive component (volatility clustering); asset returns and prices are negatively correlated with the conditional volatility (see Black 1976; Bekaert and Wu 2000); volatility is too highthat is, it is not compatible with asset pricing models (excess volatility); and volatility is linked to volume but is only in part explained by the arrival of information in the market and by macroeconomic fundamental news variables. Moreover, looking at the volatility implicit in option prices, it is observed that it is not constant as suggested by the Black and Scholes model as it depends on the asset price-strike ratio (smile effect). On these topics we refer to Ghysels, Harvey, and Renault (1996) and Bates (1995). To these problems we should add that volatility is a crucial quantity in price-hedge contingent claims and for risk management (e.g., VAR-like methodologies).

To cope with this empirical evidence, asset price evolution has been described by stochastic differential equations different from the classical Black-Scholes equation. As an example, volatility clustering has been modeled assuming an ARCH-GARCH model that is, volatility follows a stochastic process characterized by an autoregressive component (see Andersen and Bollerslev 1998). In other cases, volatility has been modeled as a level dependent quantity (i.e., an unknown time-independent function of the asset price). This type of model is included in our setting. In what follows, we present three different motivations for this type of dependence.

First of all, this way to model asset price volatility is well suited to capture the relationship between volatility and asset price return. Volatility changes are negatively correlated with stock returns and volatility decreases with the asset price. This phenomenon has been explained as evidence of a leverage effect; that is, a price drop increases financial 
leverage which makes the asset riskier, thereby inducing a higher volatility. A different interpretation relies on time-varying risk premia. On these two interpretations see Bekaert and $\mathrm{Wu}$ (2000). The simplest way to model the negative relation between asset pricereturn and volatility is to assume a constant elasticity variance model (see Cox and Ross 1976; Cox 1997; and see Example 2.4). Level dependent volatility has been also conjectured to reproduce the implied volatility smile (see Derman and Kani 1994; Dupire 1994; Hobson and Rogers 1998). A different perspective to introduce level dependent volatility is to build a model with heterogeneous agents (e.g., fundamentalist, rational, portfolio insurance traders) deriving the stochastic process for asset price in equilibrium (see Frey and Stremme 1997; Platen and Schweizer 1998).

Computation of the elasticity matrix is a three-step outcome; at each step it is necessary to compute volatilities of observed quantities or computed in a previous step. The matrix can be computed in real time by extending the methodology based on Fourier series developed in Malliavin and Mancino (2002) to compute volatility of diffusion processes. An interesting feature of the method based on Fourier series expansion is that the volatility is constructed as a function of time; this fact stabilizes the computation of the quantities needed in the computation of the matrix, because obviously the three steps entail a loss in the precision of the computation. Explicit expressions in terms of Fourier coefficients of asset prices leading to real-time determination of feedback rates are provided in Section 4.1.

We can describe our approach through a metaphor from Hamiltonian mechanics. The state of the market described by a stochastic process for asset prices is the outcome of a market equilibrium, and its evolution through time is described by correlations between price changes and instantaneous volatility changes. In Hamiltonian mechanics, principal modes of oscillation of a Hamiltonian system nearby at equilibrium are determined by the eigenvalues of the Hessian, which is the matrix given by the second derivatives of the Hamiltonian; the last two steps of our method correspond to computing the second derivatives. The elasticity matrix appears as a counterpart of the Hessian; in mechanics, the computation of second derivatives in a moving frame introduces Coriolis forces, and to work free of Coriolis forces, differentiation in an inertial Galilean frame is needed.

We present below mathematical theorems with full proofs for the construction of a hedged transfer and of the elasticity matrix. The relevance of our construction to decipher the market state is an open question, and the numerical implementation in Section 4 is intended to be a first step which needs to be followed up. A basic objection against our construction is toward the assumption that a price variation is supposed to induce immediately a volatility variation; this is not realistic as the effect needs a delay to propagate. Nevertheless the Fourier series method, which cuts down high frequency, partially takes care of this effect.

The paper is organized as follows. In Section 2 we present the univariate case together with an example (constant elasticity variance model). In Section 3 we construct the inertial frames in the multivariate case and we compute the elasticity matrix. In Section 4.1 we provide a way to compute in real time the feedback effect rate from market observations. In Section 4.2 we implement the methodology, applying it to stock and exchange rate high frequency time series.

\section{THE UNIVARIATE CASE}

Let $S(t)$ be the risky asset price at time $t$. Let us assume that $S(t)$ satisfies the following stochastic differential equation without drift:

$$
d S(t)=S(t) a_{1}(S(t)) d W(t),
$$


where $a_{1}(\cdot)$ is a deterministic function of the asset price and $W$ is a Brownian motion. Let $x(t)$ be the logarithm of the price - that is, $S(t)=\exp (x(t))$ - then $x(t)$ satisfies the stochastic differential equation

$$
d x(t)=a_{1}(S(t)) d W(t)-\frac{1}{2} a_{1}^{2}(S(t)) d t=a(x(t)) d W(t)-\frac{1}{2} a^{2}(x(t)) d t,
$$

where $a(x) \equiv a_{1}(S)=a_{1}(\exp (x))$. We assume that the function $a$ is positive and deterministic and does not depend on $t$. We assume further that $a$ belongs to $C^{2}(\mathbb{R})$.

\subsection{The Feedback Effect Rate}

The data of an infinitesimal deformation $x_{W}(t)+\varepsilon \zeta(t)$ will transform the probability measure according to the Girsanov factor

$$
\int \frac{\zeta(t)}{a(t)} d W(t)
$$

The rescaled variation is defined as

$$
z(t)=\frac{\zeta(t)}{a(t)}
$$

Then the following results hold.

THEOREM 2.1. The rescaled variation is a differentiable function with respect to $t$; its logarithmic derivative $\lambda(t)$ being called the feedback effect rate. Thus, we have

$$
z(t)=\exp \left(\int_{s}^{t} \lambda(\tau) d \tau\right) z(s), \quad s \leq t .
$$

Proof. The variational equation is given by $d \zeta(t)=a^{\prime} \zeta d W-a^{\prime} a \zeta d t$. Using Itô calculus we get

$$
\begin{gathered}
d a=a^{\prime} a d W-\frac{1}{2} a^{\prime} a^{2} d t+\frac{1}{2} a^{\prime \prime} a^{2} d t, \\
d\left(\frac{1}{a}\right)=-\frac{a^{\prime}}{a} d W+\frac{1}{2} a^{\prime} d t-\frac{1}{2} a^{\prime \prime} d t+\frac{1}{a}\left(a^{\prime}\right)^{2} d t .
\end{gathered}
$$

Therefore the rescaled variation has the following Itô differential

$$
d z(t)=\zeta(t)\left(\left(\frac{a^{\prime}}{a}-\frac{a^{\prime}}{a}\right) d W-\frac{1}{2}\left(a^{\prime}+a^{\prime \prime}\right) d t\right) .
$$

Consequently, $z(t)$ is a differentiable function of $t$ and

$$
\dot{z}(t)=-\frac{1}{2} z(t)\left(a^{\prime} a+a^{\prime \prime} a\right)=\lambda(t) z(t), \quad \lambda(t)=-\frac{1}{2}\left(a^{\prime} a+a^{\prime \prime} a\right) .
$$

Note that $\lambda=0$ in the standard Black and Scholes framework.

THEOREM 2.2. Denoting by $*$ the Itô contraction, define the following cross-volatilities:

$$
d x * d x=: A d t, \quad d A * d x=: B d t, \quad d B * d x=: C d t .
$$


Then the feedback effect rate function $\lambda$ can be expressed as

$$
\lambda=\frac{3}{8} \frac{B^{2}}{A^{3}}-\frac{1}{4} \frac{B}{A}-\frac{1}{4} \frac{C}{A^{2}} .
$$

Proof. Consider the Itô differential $d x=a d W-\frac{1}{2} a^{2} d t$; then $A=a^{2}$. Since $B$ is the cross-volatility of $A$ and $x$, it can be written as

$$
B d t=2 a a^{\prime} d x * d x=2 a^{3} a^{\prime} d t .
$$

Therefore we get

$$
a a^{\prime}=\frac{B}{2 a^{2}}=\frac{1}{2} \frac{B}{A}
$$

By definition, $C$ is the cross-volatility of $B$ and $x$, and we have $2 d\left(a a^{\prime}\right) * d x=2\left(a a^{\prime \prime}+\right.$ $\left.\left(a^{\prime}\right)^{2}\right) a^{2} d t$. On the other side we conclude from (2.2) that

$$
2 d\left(a a^{\prime}\right) * d x=\frac{1}{A^{2}}(A(d B * d x)-B(d A * d x))=\frac{1}{A^{2}}\left(A C-B^{2}\right) d t,
$$

which implies

$$
2 a a^{\prime \prime}=\frac{C}{A^{2}}-\frac{3}{2} \frac{B^{2}}{A^{3}}
$$

Thus (2.1) holds as claimed.

REMARK 2.3. As $x$ is the logarithm of a price it is dimensionless. The function $A$ appears in the parabolic operator

$$
\frac{\partial}{\partial t}+\frac{1}{2} A^{2} \frac{\partial^{2}}{\partial x^{2}}
$$

Therefore $A$ has the dimension of the inverse of time, $A \simeq T^{-1}$; by Itô calculus $d x * d x=$ $A d t$, which means that for the Itô differentiation $d \simeq T^{-\frac{1}{2}}$. We get

$$
\begin{aligned}
B d t & =d A * d x \simeq T^{-\frac{3}{2}} T^{-\frac{1}{2}}=T^{-2}, \\
\frac{B}{A} & \simeq T^{-1}, \frac{B^{2}}{A^{3}} \simeq T^{-4} T^{3}=T^{-1}, \\
C d t & =d B * d x \simeq T^{-\frac{5}{2}} T^{-\frac{1}{2}}=T^{-3}, \\
\frac{C}{A^{2}} & \simeq T^{-3} T^{2}=T^{-1} .
\end{aligned}
$$

Finally, $\lambda$ has the dimension of the inverse of a time, as it should be in the interest rate category.

EXAMPLE 2.4. We explicitly compute the rescaled variation and the instantaneous feedback rate considering a constant elasticity variance model (see Cox and Ross 1976; Cox 1997). The price process $S(t)$ satisfies

$$
d S(t)=S^{\delta}(t) d W(t),
$$

where $\delta<1$ is a fixed constant. As pointed out above, in this model volatility changes are negatively correlated with stock returns and price level. Empirical tests have shown that return distributions are less positively skewed than log-normal distribution when $\delta<1$ 
and in many cases are negatively skewed when $\delta<0$ (see Bates 1995). The basic ingredient in the definition of the rescaled variation is to use the metric defined by the volatility as the reference metric; as a result the computation of the instantaneous liquidity rate does not depend on a choice of coordinate. Taking, as in the previous paragraph, $\log S=x$ as new variable we get

$$
a(x)=\exp ((\delta-1) \log x)
$$

and

$$
\lambda=\frac{a^{2}(1-\delta)}{2 x^{2}}(x+\delta-2) .
$$

If $\delta=1$ we find the Black-Scholes model and $\lambda=0$; in the others cases the instantaneous liquidity rate changes sign at $x=2-\delta$.

\subsection{Substitution of Cross-Volatilities by Volatilities}

In Theorem 2.2 we expressed the feedback rate in terms of cross-volatilities. For a given mathematical model, it can be computed in exact terms as demonstrated in Example 3.4.

As far as the econometrical estimation of the feedback rate is concerned, crossvolatilities have the inconvenience of being related to the speed of economic propagation of feedback effects and therefore to become unstable at high frequency data.

Of course, by polarization we have the identity

$$
4 B=\operatorname{Volatility}(A+x)-\operatorname{Volatility}(A-x) .
$$

More interesting is the statement of Theorem 2.5.

\section{Theorem 2.5. Denoting}

$$
D=\text { Volatility }(\log A), \quad E=\text { Volatility }(\log D),
$$

$\lambda$ can then be expressed by the formula

$$
-4 \lambda=\eta_{1} \sqrt{D}\left(\sqrt{A}+\frac{\eta_{2}}{2} \sqrt{E}\right),
$$

where $\eta_{1}=1$ if price $x$ and volatility $A$ are positively correlated and $\eta_{1}=-1$ in the other case, and where $\eta_{2}^{2}=1$.

Proof. Starting with the identities

$$
\log A=2 \log a, \quad D=4\left(\frac{a^{\prime}}{a}\right)^{2} a^{2}=4\left(a^{\prime}\right)^{2}, \quad E=4\left(\frac{a a^{\prime \prime}}{a^{\prime}}\right)^{2}
$$

and taking square roots, we get

$$
a=\sqrt{A}, \quad a^{\prime}=\frac{\eta_{1}}{2} \sqrt{D}, \quad a a^{\prime \prime}=a^{\prime} \frac{\eta_{2}}{2} \sqrt{E} .
$$

REMARK 2.6. It is a remarkable fact that the price-volatility feedback effect can be computed effectively by measuring only a qualitative feedback, the sign of the $\eta_{i}$. 


\subsection{Stability Criteria Involving Only a One-Step Iterated Volatility}

Iterating twice the volatility operator induces numerical instability. From another point of view, whereas the rescaled variation is natural for dealing with the risk-free process, it is not appropriate for the historical price process. Of course the historical price process contains a drift that is unknown and can only be estimated, but for a short period of time, such as a single day, it can be assumed that the derivative of this drift is small and hence that the variational equation for the historical price process is the "same" as for the risk-free process.

The nonrescaled variation satisfies the equation

$$
d \zeta=a^{\prime} \zeta d W-a^{\prime} a \zeta d t .
$$

Let $\eta=\zeta^{2}$; then by Itô calculus $d \eta=\eta\left(2 a^{\prime} d W-\left(2 a^{\prime} a-\left(a^{\prime}\right)^{2}\right) d t\right)$. The condition for $\eta$ to be a supermartingale is $2 a a^{\prime} \geq\left(a^{\prime}\right)^{2}$, which is equivalent to $0 \leq a^{\prime} \leq 2 a$, or

$$
0 \leq B \leq 4 A^{2} .
$$

We can dominate $B$ by applying the Cauchy-Schwarz inequality:

$$
|B| \leq A \sqrt{D A},
$$

where $D$ denotes again the volatility of $\log A$. Thus (2.4) is guaranteed if we require

$$
0 \leq B, \quad D \leq 16 A \text {. }
$$

THEOREM 2.7. If one of the conditions (2.4), respectively (2.5), holds true then

$$
E\left[\zeta_{t}^{2}\right] \leq \zeta_{t_{0}}^{2}
$$

REMARK 2.8. Negativity of the feedback rate $\lambda$ implies a pathwise inequality, whereas (2.6) is only an inequality in the quadratic mean. Denoting by $\delta S$ the variation of the asset price $S$, we have

$$
\zeta(t)=\frac{\delta S(t)}{S(t)}
$$

\section{THE BIVARIATE CASE: ELASTICITY MATRIX}

We limit our attention to the bivariate setting. Our analysis is developed in four steps. In Section 3.1 we introduce stochastic calculus of variations in the instantaneous moving frame. In Section 3.2 we prove existence and characterize a hedged transfer of the variation of the process by requiring that its evolution be described by an ordinary differential equation. In Section 3.3 we introduce the inertial derivative using the inertial frame. In Section 3.4 we fully compute the elasticity matrix (the equivalent of $\lambda$ in the previous section).

\subsection{Stochastic Calculus of Variations in the Instantaneous Moving Frame}

We consider the process describing the evolution of the logarithm of two asset prices. We get an $\mathbb{R}^{2}$-valued process $x^{\alpha}(t), \alpha=1,2$. We assume again that there is no drift, or if 
one wants, we work under the risk-neutral probability measure with a null risk-free rate. The variance matrix is defined by the identities

$$
\mathbf{A}^{\alpha, \beta}(t) d t=d x^{\alpha}(t) * d x^{\beta}(t),
$$

where $*$ denotes Itô contraction. Note that matrix $\mathbf{A}$, which we assume to be nondegenerate, can be measured through market data. Since $\mathbf{A}$ is symmetric, it may be written as $\mathbf{A}=\mathbf{U}^{*} \mathbf{D U}$, where $\mathbf{D}$ is a strictly positive diagonal matrix and $\mathbf{U}$ is an orthogonal matrix. Define

$$
\sqrt{\mathbf{A}}=\mathbf{U}^{*} \sqrt{\mathbf{D}} \mathbf{U}=\mathbf{A}^{1 / 2} .
$$

Then $\sqrt{\mathbf{A}}$ can be determined from market data as the unique positive matrix which has A as its square. We denote by $A_{1}(t), A_{2}(t)$ the two column vectors constituting the two columns of the matrix $\sqrt{\mathbf{A}}$; they are linearly independent: at time $t, A_{1}(t), A_{2}(t)$ constitute the instantaneous frame of the market; as $\left(A_{1} A_{2}\right)$ is a symmetric matrix we have the identity $A_{1}^{2}(t)=A_{2}^{1}(t)$.

The (risk-free) measure has the following infinitesimal generator:

$$
\mathcal{L}=\frac{1}{2} \sum_{k=1,2} A_{k}^{\alpha} A_{k}^{\beta} \partial_{\alpha, \beta}^{2}+A_{0}^{\gamma} \partial_{\gamma} \quad \text { where } A_{0}^{\gamma}(t)=-\frac{1}{2} \sum_{k=1,2}\left(A_{k}^{\gamma}\right)^{2}(t) .
$$

The purpose of this paragraph is to introduce the classical stochastic calculus of variations for (3.1) in a geometric formalism which is suitable to introduce the inertial stochastic calculus of variations of the next paragraph. Our procedure of the previous section in dimension one is generalized verbatim to dimension two. See Makhankov (1997) for related geometric structures connected to volatility.

We denote by $\omega^{s}, s=1,2$, the differential forms dual to the vector fields $A_{k}:\left\langle A_{k}, \omega^{s}\right\rangle=$ $\delta_{k}^{s}$, where $\delta_{*}^{*}$ is the Kronecker symbol; denoting by $\omega$ the matrix having $\omega^{k}$ as $k$ th line we get

$$
\omega=\mathbf{A}^{-1 / 2} .
$$

The forms $\omega^{s}$ are therefore measurable by market data; their expression in coordinates is

$$
\omega^{s}=\sum_{\alpha=1,2} b_{\alpha}^{s} d x^{\alpha}
$$

The co-boundaries

$$
d \omega^{s}=\left(\frac{\partial b_{2}^{s}}{\partial x^{1}}-\frac{\partial b_{1}^{s}}{\partial x^{2}}\right) d x^{1} \wedge d x^{2}
$$

can be computed through inversion of the identities

$$
d b_{\alpha}^{s} * d x^{\beta}=\sum_{\gamma} \frac{\partial b_{\alpha}^{s}}{\partial x^{\gamma}} \mathbf{A}^{\beta, \gamma} d t \quad \text { or } \quad \frac{\partial \omega}{\partial x^{\gamma}} d t=-\frac{1}{2} \mathbf{A}^{-3 / 2} d \mathbf{A} * \mathbf{A}^{-1 / 2} d x^{\gamma} .
$$

There exist two functions $u^{s}(t), s=1,2$, called structural functions of the instantaneous frame such that

$$
d \omega^{s}=-u^{s} \omega^{1} \wedge \omega^{2}
$$

THEOREM 3.1. The structural functions $u^{s}, s=1,2$, determine the stochastic calculus of variations in the instantaneous frame; the martingale part of the variation process in the instantaneous frame is given in (3.8) below. 
Proof. The risk-free process satisfies the Itô SDE

$$
d x_{W}(t)=A_{1} d W^{1}+A_{2} d W^{2}+A_{0} d t,
$$

where $W$ is a two-dimensional Brownian motion; denote $\left.\mathcal{A}_{k}\right|_{\beta} ^{\alpha}=\partial_{\beta} A_{k}^{\alpha}$. The usual variation transfer satisfies the Itô SDE:

$$
d z_{W}=\mathcal{A}_{1}\left(z_{W}\right) d W^{1}+\mathcal{A}_{2}\left(z_{W}\right) d W^{2}+\mathcal{A}_{0}\left(z_{W}\right) d t .
$$

Writing the vector $z(t)$ in the instantaneous frame gives

$$
z(t)=\sum_{s=1,2} \zeta^{s}(t) A_{s}(t)
$$

where $\zeta^{s}(t):=\left\langle z(t), \omega^{s}(t)\right\rangle$. When computing the martingale part of the Itô differential of the right-hand side of (3.6), we get up to terms of bounded variation:

$$
\begin{aligned}
\left(\mathcal{A}_{1}\left(A_{1}\right) d W^{1}\right. & \left.+\mathcal{A}_{1}\left(A_{2}\right) d W^{2}\right) \zeta^{1}+\left(\mathcal{A}_{2}\left(A_{1}\right) d W^{1}+\mathcal{A}_{2}\left(A_{2}\right) d W^{2}\right) \zeta^{2} \\
& +A_{1} d \zeta^{1}+A_{2} d \zeta^{2} .
\end{aligned}
$$

Expressing the differential of the left-hand side of (3.6) by (3.5), the Lie bracket appears and we have

$$
\left[A_{1}, A_{2}\right]\left(\zeta^{1} d W^{2}-\zeta^{2} d W^{1}\right) \simeq A_{1} d \zeta^{1}+A_{2} d \zeta^{2},
$$

where given two semimartingales $S_{1}, S_{2}$ we write $d S_{1} \simeq d S_{2}$ for the fact that $S_{1}-S_{2}$ is a process of bounded variation. By means of the well-known identity

$$
\left\langle A_{1} \wedge A_{2}, d \omega^{s}\right\rangle+\left\langle\left[A_{1}, A_{2}\right], \omega^{s}\right\rangle=\partial_{A_{1}}\left(\left\langle\omega^{s}, A_{2}\right\rangle\right)-\partial_{A_{2}}\left(\left\langle\omega^{s}, A_{1}\right\rangle\right)
$$

as the right-hand side vanishes, we derive $\left[A_{1}, A_{2}\right]=u^{1} A_{1}+u^{2} A_{2}$.

Thus we get the martingale part of variation transfer in the instantaneous frame:

$$
d \zeta^{1} \simeq u^{1}\left(\zeta^{1} d W^{2}-\zeta^{2} d W^{1}\right), \quad d \zeta^{2} \simeq u^{2}\left(\zeta^{1} d W^{2}-\zeta^{2} d W^{1}\right)
$$

\subsection{Characterization and Existence of the Hedged Variation}

The infinitesimal Radon-Nikodym derivative effect of the (risk-free) measure of a perturbation $\zeta^{*}\left(t_{0}\right)$ up to time $T$ is given by the infinitesimal Girsanov factor

$$
\Gamma=\frac{1}{T-t_{0}} \int_{t_{0}}^{T}\left(\zeta^{1} d W^{1}+\zeta^{2} d W^{2}\right)
$$

therefore

$$
E\left(\Gamma^{2}\right)=\frac{1}{\left(T-t_{0}\right)^{2}} E\left(\int_{t_{0}}^{T}\|\zeta\|^{2} d t\right) \quad \text { where }\|\zeta\|^{2}=\sum_{s=1,2}\left(\zeta^{s}\right)^{2}
$$

DEFINITION 3.2. A hedged transfer of a perturbation is a transfer such that the variation evolution satisfies an ordinary differential equation.

From Eq. (3.8) we conclude that

$$
\frac{1}{2} d\|\zeta\|^{2} \simeq a\left(\zeta^{1} d W^{2}-\zeta^{2} d W^{1}\right) \quad \text { where } a=u^{1} \zeta^{1}+u^{2} \zeta^{2}
$$


In case $u\left(t_{0}\right) \not \equiv 0$, choosing $\zeta\left(t_{0}\right)=\zeta_{0}$ as the starting value such that $a \neq 0$, we see that the transfer along the instantaneous moving frame is not a hedged transfer.

To get a hedged transfer we need to introduce a supplementary degree of freedom by performing a properly chosen random rotation (continuously depending on time) of the instantaneous moving frame. In dimension one, the group of rotations consists of multiplication by 1 or by -1 ; hence this extra degree of freedom does not exist. We showed in Section 2 that in dimension one the transfer in the instantaneous moving frame is indeed a hedged transfer.

Denote by $\mathcal{R}_{\vartheta}$ the rotation by an angle $\vartheta$. The vector fields obtained from the fields $A_{k}$ by the rotation $\mathcal{R}_{\vartheta}$ are

$$
A_{1}^{\vartheta}=A_{1} \cos \vartheta+A_{2} \sin \vartheta, \quad A_{2}^{\vartheta}=A_{2} \cos \vartheta-A_{1} \sin \vartheta
$$

We denote by $\vartheta_{W}(t)$ the semimartingale

$$
d \vartheta_{W}(t)=\phi^{1} d W^{1}+\phi^{2} d W^{2}+\phi^{0} d t, \quad \vartheta_{W}\left(t_{0}\right)=0
$$

where the coefficients $\phi^{*}$ are semimartingales and will be determined later.

The following Itô SDE differs from the infinitesimal generator of the risk-free measure by a first-order term

$$
d x_{W}^{\vartheta}(t)=A_{1}^{\vartheta}\left(x_{W}^{\vartheta}(t)\right) d W^{1}+A_{2}^{\vartheta}\left(x_{W}^{\vartheta}(t)\right) d W^{2}+A_{0}\left(x_{W}^{\vartheta}(t)\right) d t .
$$

In fact, for the second-order terms we have $\frac{1}{2}\left(\partial_{A_{1}^{\vartheta}}^{2}+\partial_{A_{2}^{\vartheta}}^{2}\right)=\frac{1}{2}\left(\partial_{A_{1}}^{2}+\partial_{A_{2}}^{2}\right)$. We consider the new frame $A_{1}^{\vartheta}, A_{2}^{\vartheta}$. The differential forms

$$
\omega_{\vartheta}^{1}=\omega^{1} \cos \vartheta+\omega^{2} \sin \vartheta, \quad \omega_{\vartheta}^{2}=\omega^{2} \cos \vartheta-\omega^{1} \sin \vartheta
$$

constitute the dual basis; we have $\omega^{1} \wedge \omega^{2}=\omega_{\vartheta}^{1} \wedge \omega_{\vartheta}^{2}$; hence the structural equation for the new frame is given by the vector $\mathcal{R}_{\vartheta}(u)$.

THEOREM 3.3. The transfer given by (3.12) is a hedged transfer in an infinitesimal neighborhood of $t_{0}$ if and only if condition (3.13) below holds true.

Proof. The infinitesimal variation of the Brownian path $W$ is given by $W \mapsto W+\varepsilon h$ where $h \in H^{1}$ is in the Cameron-Martin space. To get the initial variation $\zeta_{0}$ we take a sequence $h_{n} \in H^{1}$ such that $\dot{h}_{n} \rightarrow \zeta_{0} \times \delta_{0}$, the Dirac mass at $t_{0}$. From (3.11) we obtain

$$
\left.\lim _{n} \frac{d}{d \varepsilon}\right|_{\varepsilon=0} \mathcal{R}_{\vartheta_{W+\varepsilon h_{n}}}=\left(\zeta_{0}^{1} \phi^{1}+\zeta_{0}^{2} \phi^{2}\right) \rho\left(\mathcal{R}_{\vartheta_{W}}\right), \quad \text { where } \rho=\left.\frac{d}{d \varepsilon}\right|_{\varepsilon=0} \mathcal{R}_{\varepsilon}
$$

Therefore the variation of $\vartheta$ induces on (3.12) for $t$ close to $t_{0}$ the variation

$$
B \rho(W(t)), \quad B=\zeta_{0}^{1} \phi^{1}+\zeta_{0}^{2} \phi^{2}
$$

Equation (3.6) holds true in the frame $\left(A_{1}^{\vartheta}, A_{2}^{\vartheta}\right)$. Taking into account that $\vartheta(t) \rightarrow 0$ as $t \searrow t_{0}$, we get for $t$ close to $t_{0}$ the variation

$$
\zeta(t)-\zeta\left(t_{0}\right) \simeq u\left(\zeta_{0}^{1} W^{2}(t)-\zeta_{0}^{2} W^{1}(t)\right)+B \rho(W(t))
$$


the condition of a hedged transfer means that the coefficients of $W^{1}(t)$ and $W^{2}(t)$ vanish in the following expression:

$$
\left(\zeta_{0}^{1} u^{1}+\zeta_{0}^{2} u^{2}\right)\left(\zeta_{0}^{1} W^{2}(t)-\zeta_{0}^{2} W^{1}(t)\right)-B \zeta_{0}^{1} W^{2}(t)+B \zeta_{0}^{2} W^{1}(t)
$$

We obtain in this way the condition $\zeta_{0}^{1} u^{1}+\zeta_{0}^{2} u^{2}=\zeta_{0}^{1} \phi^{1}+\zeta_{0}^{2} \phi^{2}=0$; this equation holding true for all initial values $\left(\zeta_{0}^{1}, \zeta_{0}^{2}\right)$ implies

$$
\phi^{1}=u^{1}, \quad \phi^{2}=u^{2} .
$$

\subsection{Inertial Frame and Inertial Derivative}

Under the hypothesis ensuring existence of a hedged transfer, the norm $\|\zeta\|$ satisfies a first-order ordinary differential equation, completely determined by an instantaneous elasticity matrix. This subsection and the next one are devoted to its computation. To this end, one approach could be, as we have done in the univariate case, to resume the steps of the last subsection, at each step the full Itô calculus being applied. This approach does not require any new concepts; from a computational point of view, however, it is relatively heavy.

We prefer a more conceptual road, based on a machinery reminiscent of celestial mechanics where the Newton principle of inertia stays valid only in Galilean framesthat is, frames having as origin the center of mass of the solar system, their axis being fixed relatively to the stars.

Given a vector field $Y$ along the trajectory we express it in the instantaneous frame $\left(A_{1}, A_{2}\right)$ as $Y=\sum_{i=1,2} \eta^{i} A_{i}$; in this way the data of a vector field are equivalent to the data of the two processes $\eta^{i}$. The inertial derivative of the vector field $Y$ is the $2 \times 2$ matrix

$$
\nabla_{k} Y=\left(\partial_{A_{k}} \eta^{1}\right) A_{1}+\left(\partial_{A_{k}} \eta^{2}\right) A_{2}-u^{k} \rho(Y), \quad k=1,2
$$

a matrix which can be measured through market data, as a matter of fact:

$$
\partial_{A_{\alpha}} \eta=\sum_{\beta=1,2}\left[\mathbf{A}^{-1 / 2}\right]_{\beta}^{\alpha}\left(d \eta * d x^{\beta}\right) .
$$

(Recall that $\rho\left(A_{1}\right)=A_{2}, \rho\left(A_{2}\right)=-A_{1}$, which follows by differentiating (3.10) with respect to $\vartheta$ at 0 .) Finally, for a vector field $Z=\sum_{i} \zeta^{i} A_{i}$ let $\nabla_{Z} Y:=\sum_{i} \zeta^{i} \nabla_{i} Y$.

Invoking the process $x^{\vartheta}$ defined by (3.12), the inertial stochastic differential is

$$
\nabla_{t}(Y)=A_{1} d \eta^{1}+A_{2} d \eta^{2}-\left(u \mid d x^{\vartheta}\right) \rho(Y)
$$

where $(u \mid v)=\sum_{i} u^{i} v^{i}$ denotes the scalar product.

THEOREM 3.4. The choice of $\vartheta$ made by imposing (3.13) implies that for $t \rightarrow t_{0}$,

$$
\nabla_{t} A_{i}^{\vartheta} \simeq 0, \quad i=1,2 .
$$

Proof. The components of the vector field $A_{1}^{\vartheta}$ in the instantaneous frame are $(\cos \vartheta, \sin \vartheta)$. Therefore the second component of $\nabla_{t} A_{1}^{\vartheta}$ is, computing $d \vartheta$ by (3.11),

$$
\begin{aligned}
\simeq & +\cos \vartheta\left(\phi^{1} d W^{1}+\phi^{2} d W^{2}\right) \\
& -\cos \vartheta\left[\left(u^{1} \cos \vartheta+u^{2} \sin \vartheta\right) d W^{1}+\left(-u^{1} \sin \vartheta+u^{2} \cos \vartheta\right) d W^{2}\right] .
\end{aligned}
$$

As $t \rightarrow t_{0}$ we get $\phi^{1}-u^{1}=0$ and $\phi^{2}-u^{2}=0$. 


\subsection{Differential Geometry in the Instantaneous Frame}

In this subsection we shall replace the computations on semimartingales by computations on their associated infinitesimal generators, leading to manipulations of PDEs familiar to differential geometers.

Given a (random) function of time $\phi(t)$, its gradient is the vector field

$$
Y(t)=\left(\partial_{A_{1}} \phi\right) A_{1}+\left(\partial_{A_{2}} \phi\right) A_{2},
$$

where the partial derivatives are implementable through (3.15); the divergence of a vector field $Z=\sum_{i} \zeta^{i} A_{i}$ is the function defined by

$$
-\left(\nabla_{1} Z\right)^{1}-\left(\nabla_{2} Z\right)^{2}=-\left(\partial_{A_{1}} \zeta^{1}+\partial_{A_{2}} \zeta^{2}\right)+\left(u^{2} \zeta^{1}-u^{1} \zeta^{2}\right)
$$

the Laplacian $\Delta \phi=-\frac{1}{2}$ div grad $\phi$ has the following expression:

$$
2 \Delta=\partial_{A_{1}}^{2}+\partial_{A_{2}}^{2}+u^{1} \partial_{A_{2}}-u^{2} \partial_{A_{1}} .
$$

Expressing $2 \Delta$ in the coordinates $x^{\alpha}$ (constituted by the logarithms of the prices) gives

$$
2 \Delta=\sum_{k, \alpha, \beta} A_{k}^{\alpha} A_{k}^{\beta} \partial_{\alpha, \beta}^{2}+\partial_{Z_{1}}, \quad Z_{1}=\left(\sum_{k, \alpha} A_{k}^{\alpha} \partial_{\alpha}\left(A_{k}^{1}\right), \sum_{k, \alpha} A_{k}^{\alpha} \partial_{\alpha}\left(A_{k}^{2}\right)\right) .
$$

Finally using (3.1) and (3.18) we get

$$
\mathcal{L}=\Delta+\partial_{\mathcal{Q}}, \quad 2 \mathcal{Q}=u^{2} A_{1}-u^{1} A_{2}-Z_{1}+2 A_{0},
$$

where $A_{0}$ has been defined in (3.1); the vector field $\mathcal{Q}$ encodes the information needed to go from finance to geometry.

We proceed with some commutation relations between geometric operators:

$$
\nabla_{1} A_{2}-\nabla_{2} A_{1}=u^{1} A_{1}+u^{2} A_{2}=\left[A_{1}, A_{2}\right] .
$$

The curvature is defined by

$$
\mathcal{C}=\left(\nabla_{1} \nabla_{2}-\nabla_{2} \nabla_{1}-u^{1} \nabla_{1}-u^{2} \nabla_{2}\right) Y .
$$

In its expression all derivatives on the components $\eta^{*}$ disappear; it therefore constitutes an endomorphism of $\mathbb{R}^{2}$. Denoting by $\varepsilon_{i}$ the basis $(1,0),(0,1)$ of $\mathbb{R}^{2}$,

$$
\nabla_{2} \varepsilon_{1}=-u^{2} \varepsilon_{2}, \quad \nabla_{1} \varepsilon_{1}=-u^{1} \varepsilon_{2}, \quad \nabla_{1} \varepsilon_{2}=u^{1} \varepsilon_{1}, \quad \nabla_{2} \varepsilon_{2}=u^{2} \varepsilon_{1} .
$$

We deduce

$$
\mathcal{C}\left(\varepsilon_{1}\right)=-R \varepsilon_{2}, \quad R=\partial_{A_{1}} u^{2}-\partial_{A_{2}} u^{1}+\|u\|^{2}, \quad\|u\|^{2}=\left(u^{1}\right)^{2}+\left(u^{2}\right)^{2} .
$$

Resuming the computation for $\varepsilon_{2}$ we obtain

$$
\mathcal{C}=-R \rho .
$$

Decompose the drift $\mathcal{Q}$ in the frame to obtain $\mathcal{Q}=c_{1} A_{1}+c_{2} A_{2}$, and denote $\mathcal{D}$ its inertial derivative in the sense of (3.14):

$$
\mathcal{D}=\left(\begin{array}{ll}
\partial_{A_{1}} c_{1}+u^{1} c_{2} & \partial_{A_{1}} c_{2}-u^{1} c_{1} \\
\partial_{A_{2}} c_{1}+u^{2} c_{2} & \partial_{A_{2}} c_{2}-u^{2} c_{1}
\end{array}\right)
$$

The elasticity matrix $\mathcal{E}$ is the following implementable symmetric $2 \times 2$ matrix:

$$
2 \mathcal{E}=-R \times \text { Identity }-\mathcal{D}-\mathcal{D}^{*} .
$$


The feedback rate of a vector $\eta=\left(\eta^{1}, \eta^{2}\right)$ at time $s$ is the homogeneous function of degree zero defined as

$$
\lambda\left(s, Y_{s}\right)=\frac{1}{\|\eta\|^{2}}\left(\mathcal{E}_{s}(\eta) \mid \eta\right), \quad Y_{s}=\eta^{1} A_{1}(s)+\eta^{2} A_{2}(s)
$$

THEOREM 3.5 (Transfer of a variation and Greeks computation). Define the angle $\vartheta$ by the following Itô SDE

$$
d \vartheta(s)=\left(\mathcal{R}_{\vartheta}(u) \mid d W\right)+\left(\mathcal{R}_{\vartheta}(u) \mid \rho(u)+\gamma\right) d t, \quad \vartheta\left(t_{0}\right)=0,
$$

where $\gamma$ is defined by the relation $\mathcal{D}-\mathcal{D}^{*}=2 \gamma \rho$. Given $t_{0}$ and a vector $Y_{t_{0}}$ at $x_{W}\left(t_{0}\right)$, define its rolling as

$$
Y_{s}=\eta^{1}(s) A_{1}(s)+\eta^{2}(s) A_{2}(s), \quad \eta(s)=\mathcal{R}_{\vartheta(s)}\left(\eta\left(t_{0}\right)\right) .
$$

The rolling-damping is defined as

$$
\tilde{Y}_{s_{0}}=\exp \left(\int_{t_{0}}^{s_{0}} \lambda\left(s, Y_{s}\right) d s\right) Y_{s_{0}} .
$$

Consider an European contingent claim $F$ with maturity $T>t_{0}$. Then we have the Greeks formula

$$
\left.\frac{d}{d \varepsilon}\right|_{\varepsilon=0} E_{x_{W}\left(t_{0}\right)+\varepsilon Y_{t_{0}}}\left[F\left(x_{W}(T)\right)\right]=E\left[\left\langle\tilde{Y}_{T}, d F\right\rangle_{x_{W}(T)}\right]=E[F \Gamma],
$$

where the weight $\Gamma$ satisfies the inequality

$$
E\left(\Gamma^{2}\right) \leq \frac{\left\|\eta\left(t_{0}\right)\right\|^{2}}{\left(T-t_{0}\right)^{2}} E\left[\int_{t_{0}}^{T} \exp \left(2 \int_{t_{0}}^{s} \lambda\left(t, Y_{t}\right) d t\right) d s\right] .
$$

Proof. See Cruzeiro and Malliavin (1998) and Fang and Malliavin (1993).

It results from (3.29) that the market is stable when the two eigenvalues of the elasticity matrix are negative.

\section{NUMERICAL IMPLEMENTATION}

In this section we come back to the one-dimensional setting of Section 2. The functions $A$, $B, C$ can be computed through the Fourier method developed in Malliavin and Mancino (2002).

\subsection{Computation of the Feedback Rate}

We assume that $x$ is measured on the time interval $[0,2 \pi]$. The Fourier coefficients of $d x$ are given by

$$
\begin{aligned}
& \alpha_{0}:=a_{0}(d x)=\frac{1}{2 \pi} \int_{0}^{2 \pi} d x(t), \\
& \alpha_{k}:=a_{k}(d x)=\frac{1}{\pi} \int_{0}^{2 \pi} \cos (k t) d x(t), \\
& \beta_{k}:=b_{k}(d x)=\frac{1}{\pi} \int_{0}^{2 \pi} \sin (k t) d x(t) .
\end{aligned}
$$


By Malliavin and Mancino (2002) we have

$$
\begin{aligned}
& a_{0}(A)=\lim _{N} \frac{\pi}{N+1-n_{0}} \sum_{s=n_{0}}^{N} \frac{1}{2}\left(\alpha_{s}^{2}+\beta_{s}^{2}\right) \\
& a_{k}(A)=\lim _{N} \frac{2 \pi}{N+1-n_{0}} \sum_{s=n_{0}}^{N} \alpha_{s} \alpha_{s+k} \\
& b_{k}(A)=\lim _{N} \frac{2 \pi}{N+1-n_{0}} \sum_{s=n_{0}}^{N} \alpha_{s} \beta_{s+k} .
\end{aligned}
$$

The reconstruction of $A$ from its Fourier coefficients is achieved by the classical FourierFéjer inversion formula:

$$
A(t)=\lim _{N} \sum_{k=0}^{N}\left(1-\frac{k}{N}\right)\left(a_{k}(A) \cos (k t)+b_{k}(A) \sin (k t)\right) .
$$

The coefficients of $a_{0}(d A), a_{k}(d A), b_{0}(d A)$ of $d A$ are then computed using integration by parts; for example,

$$
\begin{aligned}
a_{k}(d A) & =\frac{1}{\pi} \int_{0}^{2 \pi} \cos (k t) d A(t) \\
& =\frac{1}{\pi}(A(2 \pi)-A(0))+\frac{1}{\pi} k \int_{0}^{2 \pi} \sin (k t) A(t) d t \\
& =\frac{1}{\pi}(A(2 \pi)-A(0))+k b_{k}(A), \quad k \geq 1 .
\end{aligned}
$$

Next we compute the coefficients $a_{0}(B), a_{k}(B), b_{k}(B)$ of $B$. Because of $B d t=d A * d x$ we use results in Malliavin and Mancino (2002) for cross-volatilities; for example,

$$
\begin{aligned}
a_{k}(B) & =\lim _{N} \frac{2 \pi}{N+1-n_{0}} \sum_{s=n_{0}}^{N} \frac{1}{2}\left(a_{s}(d A) a_{s+k}(d x)+a_{s}(d x) a_{s+k}(d A)\right) \\
& =\lim _{N} \frac{2 \pi}{N+1-n_{0}} \sum_{s=n_{0}}^{N} \frac{1}{2}\left(s b_{s}(A) \alpha_{s+k}+(s+k) \alpha_{s} b_{s+k}(A)\right) \\
& =\lim _{N} \lim _{M} \frac{2 \pi}{N+1-n_{0}} \frac{2 \pi}{M+1-m_{0}} \sum_{s=n_{0}}^{N} \sum_{r=m_{0}}^{M} \frac{1}{2} \alpha_{r}\left(s \beta_{r+s} \alpha_{s+k}+(s+k) \alpha_{s} \beta_{r+s+k}\right) .
\end{aligned}
$$

The coefficients of $d B$ are then obtained by integration by parts. Finally, the computation of the coefficients of $C$ is done in an analogous way. Since $C d t=d B * d x$, we use again the results in Malliavin and Mancino for cross-volatilities.

\subsection{Estimation of $\lambda$ for Market Data}

Estimating $\lambda$ on market data can be of great interest, since the theory suggests that the sign of $\lambda$ is associated with stability of the market. A negative $\lambda$ would witness a period of stability; a positive $\lambda$ would signal instability. 
An estimate of $\lambda$ can be achieved via the Fourier expansions explained in Section 4.1. Implementing these formulas, two practical problems are in order: first, the Fourier coefficients are given in the form of limits, so that we have to replace them with finite sums; second, the price process is not observed continuously. To recover the continuous-time price from discrete observations, several interpolation schemes have been adopted in the literature (see Barucci and Renò 2000). We will adopt the previous-tick interpolation scheme (stepwise function), which has been shown, in a Monte Carlo setting, to perform better than the linear interpolation scheme.

Precise estimation of quadratic and higher order variations asks for huge quantities of data; high-frequency data are a natural candidate for this purpose. We used two data sets: a data set containing quotes of the DEM-USD foreign exchange rates from
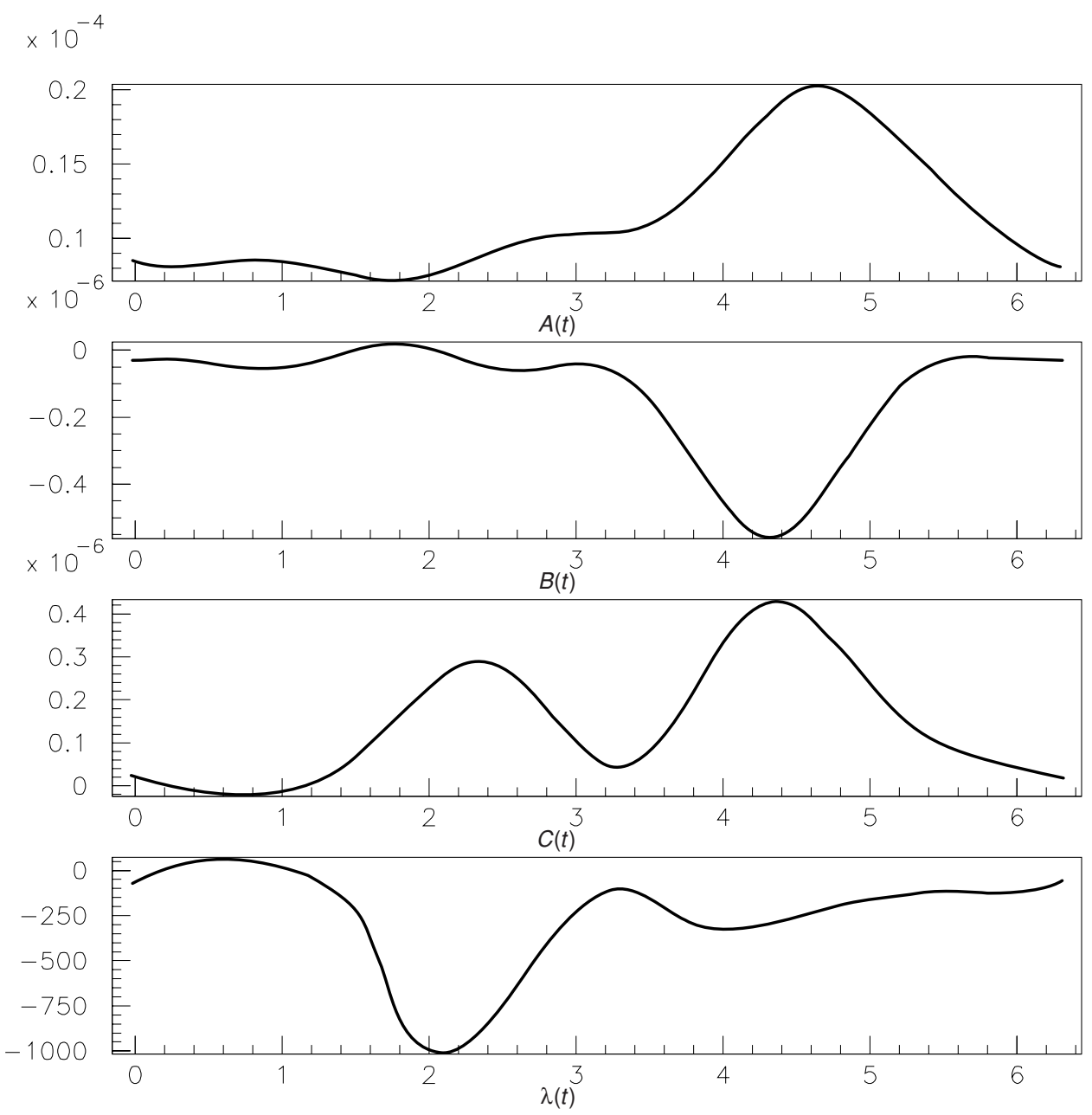

FIgURE 4.1. Average estimate of daily $A(t), B(t), C(t)$ on DEM-USD data, and corresponding value of $\lambda(t)$. On the $x$ axis, the time window $[0,2 \pi]$ corresponds to one trading day (24 hours, starting at 21:00 GMT). $A(t)$ displays a three-peak structure, each peak corresponding to the opening of major markets (Asian, European, North American). 


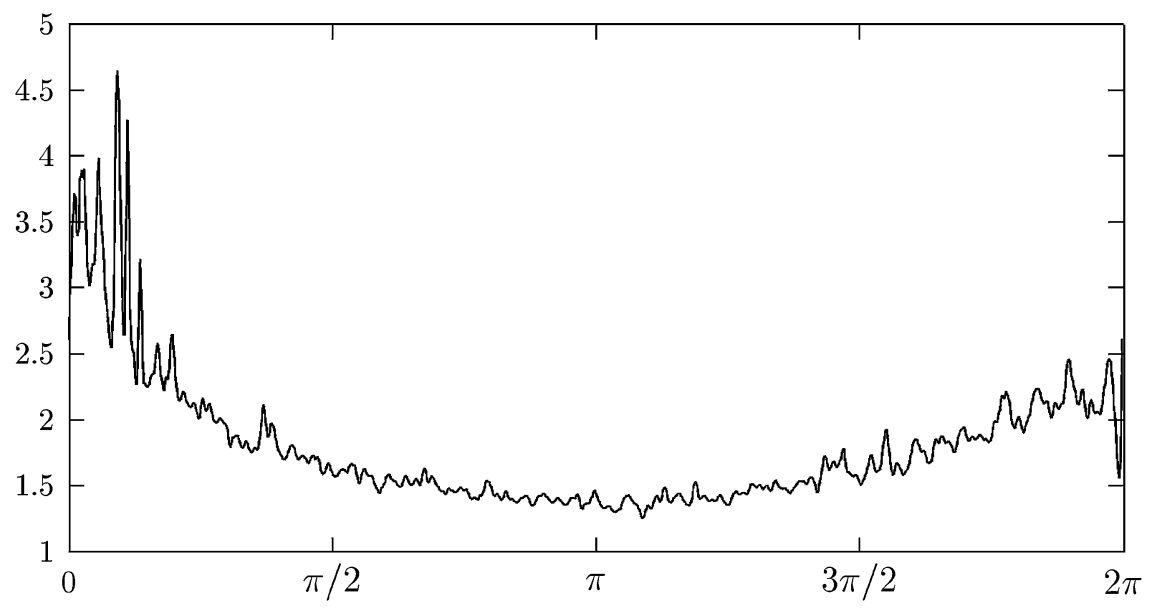

FIgURE 4.2. Average estimate of daily volatility $A(t)$ on IBM data. On the $x$ axis, the time window $[0,2 \pi]$ corresponds to one trading day (6.5 hours). $A(t)$ displays the typical $U$-shape of volatility in stock markets.

October 1992 to September 1993, and a data set of IBM quotes from January to December 1999. ${ }^{1}$

Estimation of $A(t), B(t), C(t)$ can be accomplished using the formulas in Section 4.1, after truncating them to an empirically chosen order. We have two expansions to truncate: the first is that relating the coefficients of $A, B, C$ to the coefficients of $d x$; the second one is the inversion formula that provides $A, B, C$ from their coefficients. Computation of the coefficients of $d x$ must be stopped to an empirically chosen frequency (see Barucci and Renò 2000 for a discussion of this point). For instance, computing them for frequencies larger than the inverse of the smallest distance between adjacent observations induces aliasing effects.

In the case of foreign exchange rates, estimates of $A(t), B(t), C(t)$ have been computed for a one-day time window ( 24 hours). We used 500 coefficients of $d x$ for the DEM-USD exchange rate; in the inversion formula only 20 coefficients of $A, 10$ of $B$, and 5 of $C$ are used (leading to relatively smooth curves).

We summarize the computations by averaging over the full sample for illustrative purposes.

Figure 4.1 reports the average estimate of $A(t), B(t), C(t)$ obtained with this technique, plus the corresponding value of $\lambda(t)$ obtained by equation (2.1). $A(t)$ is linked to the intraday volatility, which has been widely documented to display deterministic patterns.

Looking at Figure 4.1 we observe a three-peak structure in $A(t)$; the three peaks correspond, respectively, to the opening of Asian markets, European markets, and NorthAmerican markets; the latter peak is the largest, as expected.

In the case of the IBM stock price, estimates of $A(t), B(t), C(t)$ have been computed for a one-day time window (6.5 hours); the larger number of Fourier coefficients produces a higher resolution of the plots. Figure 4.2 gives the volatility $A(t)$ averaged over the full year; here we recognize the $U$-shaped pattern which is typical of stock market intraday volatility.

\footnotetext{
${ }^{1}$ We acknowledge Olsen \& Associates for the provision of this data set.
} 

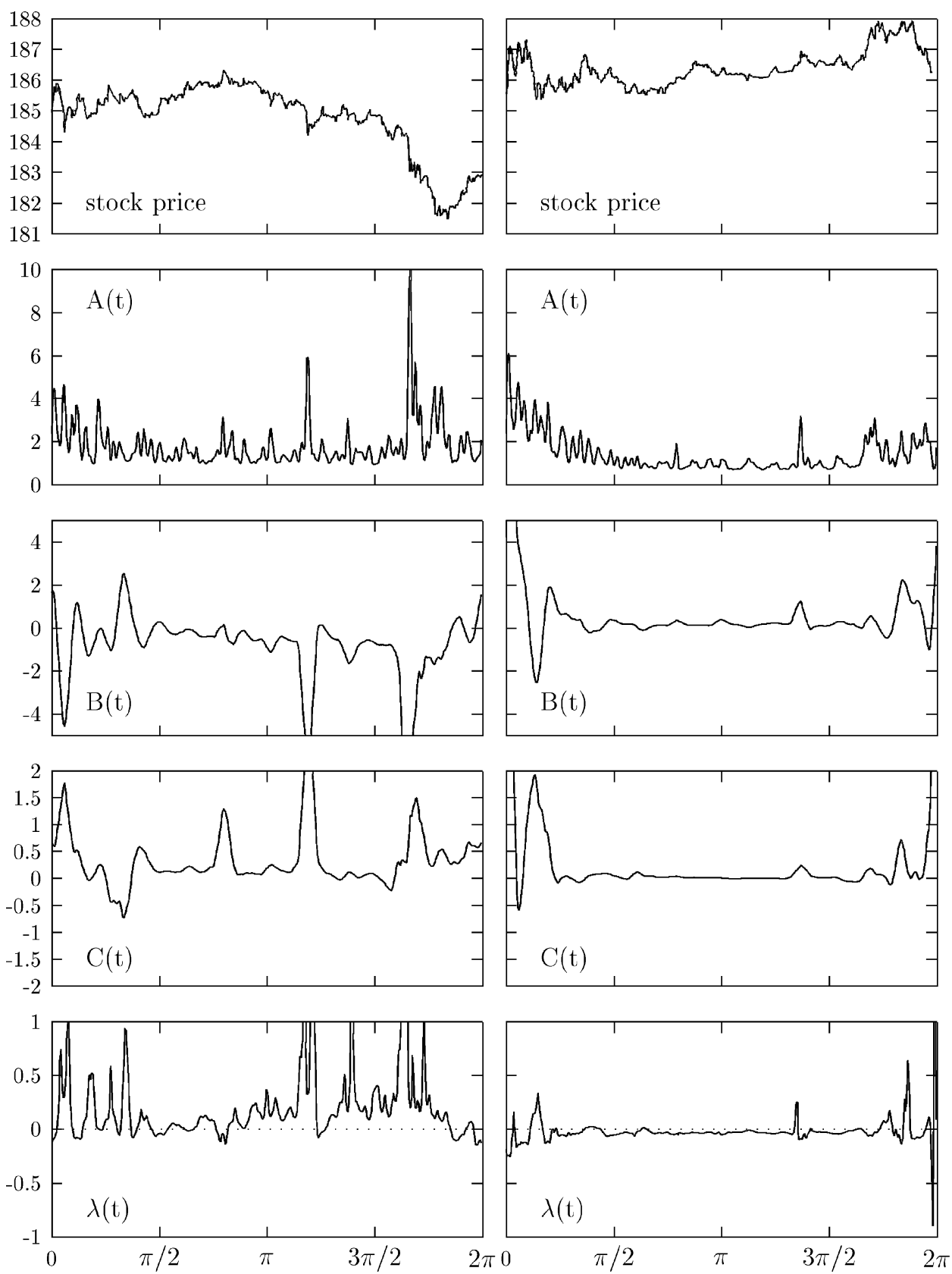

Figure 4.3. Daily values of $A(t), B(t), C(t), \lambda(t)$ on IBM data. The time windows $[0,2 \pi]$ correspond to two typical trading days in 1999 (6.5 hours each). January 4 (the left-hand side) displays positivity of $\lambda$ with large picks of $A$ (instable market); April 9 (the right-hand side) displays small and mainly negative values of $\lambda$ along with a progressive damping of $A$ (stable market). During the first two hours of trading, $A$ has about the same shape on both days, but $\lambda$ develops dramatically different shapes; computing $\lambda$ in real time could give an indicator forecasting market instability. 
Short-horizon estimates of $\lambda$ are the most important for traders. We present in Figure 4.3 as typical sample of daily (nonaveraged) estimates the values for $A(t), B(t), C(t)$, and $\lambda(t)$ for two days in 1999 .

It is noteworthy that taking the logarithm of the stock price mainly changes the scales of $A(t), B(t)$, and $C(t)$, but lets the shapes of the curves remain more or less invariant. For this reason, in Figures 4.2 and 4.3 the scales have been chosen according to the stock price (without taking logarithms).

On January 4, 1999, the beginning of the trading day reveals positivity of $\lambda$ which detects instability of the market and which is revealed by subsequent large picks of $A$. Over the whole day the positive values of $\lambda$ dominate and indicate an unstable trading day. On April 9, 1999, the beginning of the day reveals negativity of $\lambda$, which indicates stability of the market and which is revealed by a subsequent progressive damping of $A$. In contrast to January 4 , over the whole trading day, small and mainly negative values of $\lambda$ dominate and indicate stability.

As argued in this paper, precise estimation of $\lambda$ could, in principle, result in important consequences for trading strategies. In this context, it can be of some help to analyze the sign of $\lambda$ week-by-week or even day-by-day; our results show that, by using high-frequency data, an estimate of these effects can be readily accomplished.

\section{REFERENCES}

Andersen, T., and T. Bollersley (1998): Answering the Skeptics: Yes, Standard Volatility Models Do Provide Accurate Forecasts, Inter. Econ. Rev. 39, 885-905.

BARUCCI, E., and R. ReNÒ (2002): On Measuring Volatility and the GARCH Forecasting Performance, J. Inter. Financial Mkts., Inst. Money 12, 183-200.

BARucci, E., and R. Renò (2002): On Measuring Volatility of Diffusion Processes with High Frequency Data, Econ. Lett. 74, 371-378.

BAtes, D. (1995): Testing Option Pricing Models. Working paper, NBER no. 5129.

Bekaert, G., and G. Wu (2000): Asymmetric Volatility and Risk in Equity Markets, Rev. Financial Stud. 13, 1-42.

Black, F. (1976): Studies of Stock Price Volatility Changes. Proceedings of the 1976 Meetings of the American Statistical Association, 177-181.

Cox, J. C. (1997): The Constant Elasticity of Variance Option Pricing Model, J. Portfolio Mgmt. 23(3), 15-17.

Cox, J. C., and S. Ross (1976): The Valuation of Options for Alternative Stochastic Processes. J. Financial Econ. 3, 145-166.

Cruzeiro, A. B., and P. Malliavin (1998): Non Perturbative Construction of Invariant Measure through Confinement by Curvature, J. Math. Pures Appl. 77, 527-537.

Derman, E., and I. KANi (1994): Riding on the Smile, RISK 7, 32-39.

DuPIRE, B. (1994): Pricing with a Smile, RISK 7, 18-20.

FAnG, S., and P. Malliavin (1993): Stochastic Analysis on the Path Space of a Riemannian Manifold. I: Markovian Stochastic Calculus, J. Funct. Anal. 118, 249-274.

Frey, R., and A. Stremme (1997): Market Volatility and Feedback Effects from Dynamic Hedging, Math. Finance 7, 351-374.

Friedman, M. (1953): The Case of Flexible Exchange Rates; in Essays in Positive Economics, M. Friedman, ed. Chicago: University of Chicago Press, 157-203. 
Ghysels, E., A. C. Harvey, and E. Renault (1996): Stochastic Volatility; in Handbook of Statistics, vol. 14, G. S. Maddala and C. R. Rao, eds. Amsterdam: North-Holland, 119-191.

Hobson, D. G., and L. C. G. Rogers (1998): Complete Models with Stochastic Volatility, Math. Finance 8, 27-48.

Maknankov, V. G. (1997): Stochastic Differential Geometry in Finance Studies; in Nonlinear Dynamics, Chaotic and Complex Systems, E. Infeld, R. Zelacny, and A. Galkowski, eds. Cambridge, U.K.: Cambridge University Press, 296-320.

Malliavin, P., and M. E. Mancino (2002): Fourier Series Method for Measurement of MultiVariate Volatilities, Finance Stoch. 6, 49-61.

Platen, E., and M. Schweizer (1998): On Feedback Effects from Hedging Derivatives, Math. Finance 8, 67-84. 